\title{
Bosonization in $d=2$ from finite chiral determinants with a Gauss decomposition
}

\author{
A. N. Theron \\ Theory Division, CERN, CH-1211 Geneve 23, Switzerland \\ and Institute of Theoretical Physics, University of Stellenbosch, 7600 Stellenbosch, South Africa \\ F. G. Scholtz \\ Institute of Theorectical Physics, University of Stellenbosch, 7600 Stellenbosch, South Africa \\ H. B. Geyer \\ Institute of Theoretical Physics, University of Stellenbosch, 7600 Stellenbosch, South Africa \\ and Fachbereich Physik, Universität Siegen, Postfach 101240, D-57068 Siegen, Germany
}

(Received 5 June 1997)

\begin{abstract}
We show how to bosonize two-dimensional non-Abelian models using finite chiral determinants calculated from a Gauss decomposition. The calculation is quite straightforward and hardly more involved than for the Abelian case. In particular, the counterterm $A \bar{A}$, which is normally motivated from gauge invariance and then added by hand, appears naturally in this approach. [S0556-2821(97)01622-6]
\end{abstract}

PACS number(s): 11.15.Tk

The path integral approach to bosonization of twodimensional field theories was developed some time ago [1] by exploiting the fact that gauge fields may be decoupled from the fermions by making local chiral transformations. It is crucial in this approach that the anomalous contributions from the fermionic measure under chiral rotations are properly taken into account.

More recently an approach to bosonization with path integrals, referred to as smooth bosonization, was introduced [2]. Here a chiral gauge symmetry is first introduced, and the bosonization rules are then obtained by choosing an appropriate gauge. Again the anomalous contribution from the path integral measure is of great importance. For an infinitesimal chiral transformation this contribution may be calculated by the method of Fujikawa [3], but for the applications mentioned above it is necessary to obtain the anomalous Jacobian for a finite chiral transformation. One therefore requires the iteration of the infinitesimal anomaly to obtain the Jacobian for a finite chiral change of variables.

For the Abelian case this problem is rather simple, and the Abelian chiral anomaly is easily integrated to yield the free scalar boson action [1]. The non-Abelian case is, however, complicated and it is difficult to show directly that the nonAbelian anomaly can be integrated to yield the WessZumino-Witten (WZW) [4] action. In this paper we present a simple and direct derivation of this result for $\mathrm{su}(2)$, hardly more involved than the Abelian case.

Although it is well known that the finite chiral transformation yields the WZW action (or the gauged WZW action in the presence of a background field) [5], this result is of such importance in the path integral bosonization program that we consider it worthwhile to present an alternative derivation. The present derivation is not only more directly constructive than the conventional one, which proceeds indirectly by showing that the variation of the WZW model is in agreement with the anomaly [5], but it also serves to clarify some issues connected with regularization. In this context it is often stated that it is necessary to introduce a counterterm of the type $A \bar{A}$ by hand as to ensure gauge invariance $[5,6]$. We show here that this term arises naturally when the covariant form of the anomaly is iterated.

The Jacobian associated with the final chiral transformation is calculated by using the Gauss decomposition for the group. The calculation is then relatively simple and the iteration of the infinitesimal result is hardly more involved than the Abelian case. Normally there is very little advantage in writing the WZW model using an explicit parametrization for the group, because the action is complicated and the global symmetries are obscured, but in the Gauss decomposition the WZW model assumes a remarkably simple form which is closely related to the Wakimoto free field realization [7] of Kac-Moody algebras, as initially noted in Ref. [8].

We start with the current-current generating functional of free Dirac fermions:

$$
\begin{aligned}
Z_{\mathrm{F}}[A]= & \int D \bar{\psi} D \psi \exp \left(-\int d^{2} x\left[\psi_{1}^{\dagger} i \partial \psi_{1}+\psi_{2}^{\dagger} i \bar{\partial} \psi_{2}\right.\right. \\
& \left.\left.-\psi_{1}^{\dagger} A \psi_{1}-\psi_{2}^{\dagger} \bar{A} \psi_{2}\right]\right),
\end{aligned}
$$

where $A=A^{a} T^{a}, T^{a}=\frac{1}{2} \sigma^{a}$, and $\sigma^{a}$ are the Pauli spin matrices. The overbar denotes the antiholomorphic components. The source $A$ for the currents may also be viewed as a background gauge field.

We restrict ourselves to two fermion colors only, so that we can view the fermions to be in the fundamental representation of $\mathrm{SU}(2)$. Actually we have a U(2) symmetry, but we shall focus attention on the su(2) subalgebra.

In many applications it is possible to choose the light cone gauge, so that only one component of the vector $A$ is present, but we shall be more general for the moment.

We find it convenient to introduce the Cartan-Weyl basis

$$
\begin{aligned}
& T^{+}=T^{1}+i T^{2}, \\
& T^{-}=T^{1}-i T^{2},
\end{aligned}
$$




$$
T^{0}=T^{3}
$$

so that we are in fact considering the complexification of $\mathrm{su}(2)$. In this basis we denote the components of the gauge fields by $A^{ \pm}, A^{0}$. It has been noted [9] that it is convenient to consider the complexified group when considering path integral bosonization, and the basis (2) is useful in establishing a connection with the Wakimoto realization.

We now calculate the anomaly resulting from the following change of variables on the fermions,

$$
\begin{gathered}
\psi_{1} \rightarrow \psi_{1}^{\prime}=g \psi_{1}, \quad \psi_{1}^{\dagger} \rightarrow \psi_{1}^{\dagger \prime}=\psi_{1}^{\dagger} g^{-1}, \\
\psi_{2} \rightarrow \psi_{2}^{\prime}=\psi_{2}, \quad \psi_{2}^{\dagger} \rightarrow \psi_{2}^{\dagger \prime}=\psi_{2}^{\dagger} .
\end{gathered}
$$

If $g$ is infinitesimal, $g(\epsilon)=e^{i \epsilon T^{a} \theta^{a}}$, and we have the familiar anomalous contribution

$$
J_{\epsilon}=\frac{\epsilon}{\pi} \int d^{2} x \operatorname{Tr}(\theta F),
$$

with $F=\epsilon_{\mu \nu} F^{\mu \nu}$ the dual field strength and $\theta=\theta^{a} T^{a}$. The Jacobian (4) originates from the noninvariance of the path integral measure under the chiral rotation, as is most naturally established using the methods of Fujikawa [3]. We prefer the above covariant form of the anomaly, as its iterated finite form will be the gauged WZW model.

When making the finite rotation (3), we change the external gauge field used in regulating the measure, so that the anomaly associated with the finite rotation is given by

$$
\begin{aligned}
J= & \frac{1}{\pi} \int d^{2} x \int_{0}^{1} d \epsilon \operatorname{Tr}\left\{i \theta \bar{\partial}\left(g^{-1}(\epsilon) A g(\epsilon)-g^{-1}(\epsilon) i \partial g(\epsilon)\right)\right. \\
& \left.-\partial \bar{A}-\left[g^{-1}(\epsilon) A g(\epsilon)-g^{-1}(\epsilon) i \partial g(\epsilon), \bar{A}\right]\right\} .
\end{aligned}
$$

This expression is nothing but the gauged WZW action

$$
\begin{aligned}
S_{\mathrm{WZW}}= & -S[g]-\frac{i}{4 \pi} \int d^{2} x\left\{\operatorname{Tr}\left[A\left(\partial_{-} g\right) g^{-1}\right]\right. \\
& \left.+\operatorname{Tr}\left(\bar{A} g^{-1} \partial_{+} g\right)+A g A_{-} g^{-1}+A \bar{A}\right\}
\end{aligned}
$$

with

$$
\begin{aligned}
S[g]= & \frac{1}{8 \pi} \int d^{2} x \operatorname{Tr}\left(g \partial_{\mu} g^{-1} g \partial_{\mu} g^{-1}\right) \\
& +\frac{1}{12 \pi} \int_{\Gamma} d^{3} x \epsilon_{\mu \nu \rho} \operatorname{Tr}\left(g \partial_{\mu} g^{-1} g \partial_{\nu} g^{-1} g \partial_{\rho} g^{-1}\right),
\end{aligned}
$$

as we demonstrate explicitly below.

If we would have worked in the light cone gauge $\bar{A}=0$, very often the preferred gauge when two-dimensional gauge theories are studied, we would have obtained instead

$$
J=\frac{1}{\pi} \int d^{2} x \int_{0}^{1} d \epsilon \operatorname{Tr}\left\{i \theta \bar{\partial}\left(g^{-1}(\epsilon) A g(\epsilon)\right)\right\}
$$

This action is expression (6) with $\bar{A}=0$. For transparency the discussion below is restricted to this case. Results for the general case follow without any complications, as we also elaborate below.
A direct evaluation of the epsilon integration in Eq. (5) is generally not feasible. However, by first introducing the Gauss decomposition for SU(2) these integrations can easily be done. We therefore write, for the group element $g$,

$$
g=e^{i T^{+} \theta^{-}} e^{i T^{0} \theta^{0}} e^{i T^{-} \theta^{+}} .
$$

For a general element of SL(2,C) the $\theta^{a}$ are all complex, but for the identification of the Wakimoto realization we consider the transformation (9) with $\theta^{0}$ real and $\theta^{-}$the complex conjugate of $\theta^{+}$.

The transformation (3) is now performed in three steps. First we make a $T^{+}$rotation, then a $T^{0}$ rotation, and finally a $T^{-}$transformation. We therefore start by performing the local chiral transformation

$$
\begin{gathered}
\psi_{1} \rightarrow e^{i T^{+} \theta^{-}} \psi_{1}, \quad \psi_{2} \rightarrow \psi_{2}, \\
\psi_{1}^{\dagger} \rightarrow \psi_{1}^{\dagger} e^{-i T^{+} \theta^{-}}, \quad \psi_{2}^{\dagger} \rightarrow \psi_{2}^{\dagger} .
\end{gathered}
$$

The Lagrangian of Eq. (1) (with $\bar{A}=0$ ) changes to

$$
\mathcal{L} \rightarrow \mathcal{L}_{\mathrm{F}}-\psi_{1}^{\dagger} A^{\prime} \psi_{1}
$$

under transformation (10). Here $\mathcal{L}_{\mathrm{F}}$ is the Lagrange density for free Dirac fermions and

$$
\begin{aligned}
A^{\prime}= & -\partial \theta^{-} T^{+}+A^{-} T^{+}+A^{0}\left(T^{0}+i T^{+} \theta^{-}\right) \\
& +A^{+}\left[T^{-}-2 i \theta^{-} T^{0}+\left(\theta^{-}\right)^{2} T^{+}\right] .
\end{aligned}
$$

For the transformation

$$
\psi_{1} \rightarrow e^{i T^{+} \theta^{-}} \psi_{1},
$$

the contribution from the measure is obtained by substituting $g(\epsilon)$ by $e^{i \epsilon T^{+} \theta^{-}}$in expression (8). After evaluating the trace we have the following anomalous contribution to the action

$$
S_{+}=\frac{1}{\pi} \int d^{2} x \theta^{-} \overline{\partial A}{ }^{+}
$$

Terms that depend on $\epsilon$ vanish when the trace is taken, so that the $\epsilon$ integration yields one.

The second step is to perform the change of variables

$$
\begin{gathered}
\psi_{-} \rightarrow e^{i T^{0} \theta^{0}} \psi_{-}, \\
\psi_{-}^{\dagger} \rightarrow \psi_{-}^{\dagger} e^{-i T^{0} \theta^{0}} .
\end{gathered}
$$

The Lagrangian density now becomes

$$
\mathcal{L} \rightarrow \mathcal{L}_{\mathrm{F}}-\psi_{1}^{\dagger} A^{\prime \prime} \psi_{1},
$$

where

$$
\begin{aligned}
A^{\prime \prime}= & \partial \theta^{0} T^{0}-\left(\partial \theta^{-} T^{+}\right) e^{-i \theta^{0}}-\left\{\left[A^{-}+i \theta^{-} A^{0}\right.\right. \\
& \left.+A^{+}\left(\theta^{-}\right)^{2}\right] T^{+} e^{-i \theta^{0}}+A^{0} T^{0}-2 i \theta^{-} A^{+} T^{0} \\
& \left.+A^{+} T^{-} e^{i \theta^{0}}\right\} .
\end{aligned}
$$

For this transformation the contribution from the measure may again be evaluated from Eq. (8) by taking 
$g(\epsilon)=e^{i \epsilon \theta^{0} T^{0}}$, but $A$ has to be replaced by the $A^{\prime}$ of Eq. (13). The result after the trace has been performed is the additional contribution

$$
\begin{aligned}
S_{0} & =\frac{1}{2 \pi} \int d^{2} x \int_{0}^{1} d \epsilon \theta^{0} \bar{\partial}\left\{\epsilon \partial \theta^{0}+\bar{\partial} A^{0}-2 i \theta^{-} A^{+}\right\} \\
& =\frac{1}{2 \pi} \int d^{2} x\left\{\frac{1}{2} \theta^{0} \bar{\partial} \partial \theta^{0}+\theta^{0} \bar{\partial} A^{0}-2 i \theta^{0} \theta^{-} A^{+}\right\} .
\end{aligned}
$$

Finally the change of variables

$$
\psi_{-} \rightarrow e^{i T^{-} \theta^{+}} \psi_{-}
$$

is performed. We repeat the above step for $T^{-}$, taking into account that the background field $A$ has now been altered by both the $T^{+}$and $T^{0}$ rotations, and that $A^{\prime \prime}$ of expression (18) has to be used instead. This gives the final contribution

$$
\begin{aligned}
S_{-}= & \frac{1}{\pi} \int d^{2} x\left\{\theta ^ { + } \overline { \partial } \left(\partial \theta^{-} e^{-i \theta^{0}+e^{-i \theta^{0}}\left[A^{-}+i \theta^{-} A^{0}\right.}\right.\right. \\
& \left.\left.\left.+A^{+}\left(\theta^{-}\right)^{2}\right]\right)\right\} .
\end{aligned}
$$

As for the $T^{+}$transformation the integration over $\epsilon$ is trivial. The Lagrangian is now

$$
\mathcal{L}=\mathcal{L}_{\mathrm{F}}+\psi_{1}^{\dagger}\left(g^{-1} i \partial g\right) \psi_{1}-\psi_{1}^{\dagger} g^{-1} A g \psi_{1},
$$

where $g$ was given in Eq. (9)

The total anomalous contribution from the measure to the action follows from expressions (15), (19), and (21) as

$$
\begin{aligned}
& S=\frac{1}{\pi} \int d^{2} x\left\{\frac{1}{4} \partial \theta^{0} \bar{\partial} \theta^{0}+\bar{\partial} \theta^{+} e^{-i \theta^{0}} \partial \theta^{-}+A^{+}\left[\bar{\partial} \theta^{-}-i \theta^{-} \bar{\partial} \theta^{0}\right.\right. \\
& \left.+e^{-i \theta^{0}} \bar{\partial} \theta^{+}\left(\theta^{-}\right)^{2}\right]+A^{0}\left(\frac{1}{2} \bar{\partial} \theta^{0}+i \theta^{-} \bar{\partial} \theta^{+} e^{-i \theta^{0}}\right) \\
& \left.+A^{-} \bar{\partial} \theta^{+} e^{-i \theta^{0}}\right\} \text {. }
\end{aligned}
$$

As has been explicitly verified [8], this action is nothing but the WZW action when $g$ is parametrized by the Gauss decomposition (9):

$$
S=S[g]+\frac{i}{\pi} \operatorname{Tr}\left(A g \bar{\partial}^{-1}\right) .
$$

Use of expression (5) rather than (8) would have resulted in the full gauged WZW model (6). This may readily be verified. The $A \bar{A}$ terms which do not contain $g$ coupling originate from the $e^{i T^{0} \theta^{0}}$ rotation. The general form of Eq. (19) with $\bar{A} \neq 0$ follows by substituting Eq. (13) into (5), which gives, after performing the trace, additional terms such as

$$
\frac{1}{2 \pi} \int_{0}^{1} d \epsilon \theta^{0} \bar{A}^{-} A^{+} e^{\epsilon \theta^{0}}=\frac{1}{2 \pi}\left(e^{\theta^{0}}-1\right) \bar{A}^{-} A^{+} .
$$

Thus the $\epsilon$ integral gives an $A \bar{A}$ term without any $\theta$ coupling. It is therefore not necessary to view this term as a counterterm that has to be added by hand to insure gauge invariance
$[5,6]$. Since we are using a covariant form of the infinitesimal anomaly (4), the iterated anomaly is expected and indeed found to be the gauge-invariant expression (6).

The bosonic Lagrangian (23), or (24), is equivalent to that of the free fermionic model (1), as originally established by Witten [4], and normally referred to as non-Abelian bosonization. The result (23) plays an essential role in the path integral derivation of this equivalence [10].

Here we note that expression (23) is also closely linked to the Wakimoto [7] or free field realization of Kac-Moody algebras. This correspondence is the field theoretical analogue of the relation between the path integral for spin and the Dyson mapping as has been discussed in Ref. [11].

To obtain the current-current generating functional in terms of bosonic variables [10] we have to integrate the action (23) with respect to the Haar measure which reads $D \theta^{+} D \theta^{0} D \theta^{-} \operatorname{det}\left(e^{-i \theta^{0}}\right)$, in the Gauss decomposition,

$$
Z_{\mathrm{B}}[A]=\int D \theta^{+} D \theta^{0} D \theta^{-} \operatorname{det}\left(e^{-i \theta^{0}}\right) e^{-k S} .
$$

Here we allowed for a slight generalization by introducing the constant $k$. For one fermion flavor, as we have here, $k=1$, but for $k$ flavors the anomaly is yielded $k$ times so that the WZW action occurs with a multiplicative constant $k$. We also remark that the fermion currents with $k$ flavors close on a Kac-Moody algebra of level $k$.

As noted in [8], the expressions to which the $A$ currents couple in Eq. (23) are closely related to the Wakimoto realization $[7,13,14]$. To make the association explicit, we have to perform a change of variables

$$
\beta=k \bar{\partial} \theta^{+} e^{-i \theta^{0}} .
$$

Naively the Jacobian of this transformation is

$$
J_{\beta}=\frac{1}{\operatorname{det}\left(e^{-i \theta^{0}}\right)} \frac{1}{\operatorname{det}(\bar{\partial})}
$$

in which case this determinant cancels the one from the invariant group measure, with the result that we obtain a flat measure. The generating functional becomes

$$
Z_{\mathrm{B}}[A]=\int D \beta D \theta^{0} D \theta^{-} \operatorname{det}(1 / \bar{\partial}) e^{-S},
$$

with

$$
\begin{aligned}
S= & \frac{1}{\pi} \int d^{2} x\left[\frac{k}{4} \partial \theta^{0} \bar{\partial} \theta^{0}+\beta \partial \theta^{-}-i A^{+}\left[i k \bar{\partial} \theta^{-}+k \theta^{-} \bar{\partial} \theta^{0}\right.\right. \\
& \left.\left.+i \beta\left(\theta^{-}\right)^{2}\right]-i A^{0}\left(\frac{i k}{2} \bar{\partial} \theta^{0}-\theta^{-} \beta\right)-i A^{-}(i \beta)\right] .
\end{aligned}
$$

The change of variables (27) transforms the WZW action to that of free bosonic fields (30). For this reason Eq. (30) is called a free field realization of the WZW model. The expressions to which the sources $A$ couple in (30), may be viewed as a kind of "classical" Wakimoto realization [12], where corrections due to normal ordering have been neglected. This issue has partially been discussed in Ref. [8], where it was pointed out that there are corrections from the 
measure when we perform transformation (27). These corrections in particular modify the coefficient of the $\partial \theta^{0} \bar{\partial} \theta^{0}$ term. Although a complete derivation is still lacking, one expects similar corrections to the above classical Wakimoto currents to obtain the correct expressions

$$
\begin{gathered}
J^{-}=i k \bar{\partial} \theta^{-}+\sqrt{2} \sqrt{(k+2)} \theta^{-} \bar{\partial} \theta^{0}+i \beta\left(\theta^{-}\right)^{2}, \\
J^{0}=\frac{i \sqrt{(k+2)}}{\sqrt{2}} \bar{\partial} \theta^{0}-\theta^{-} \beta, \\
J^{+}=i \beta .
\end{gathered}
$$

The charge at infinity may similarly be understood as originating from such anomalous corrections in the path integral approach [8].
In conclusion, we have presented a simple and systematic procedure to evaluate finite chiral determinants, leading to a constructive identification of the counterterm $A \bar{A}$. This procedure also facilitates the identification of a free field or Wakimoto realization of Kac-Moody algebras. A constructive procedure to deal with the quantum corrections to these currents is, however, still lacking, a not uncommon situation when general changes of variables are performed in the path integral setting.

We acknowledge financial support by the Foundation for Research Development. N.T. would like to thank the Theory Division at CERN for their warm hospitality. H.B.G. also acknowledges support by the Alexander von Humboldt Stiftung and would like to thank Gottfried Holzwarth and Hans Walliser for their hospitality at the University of Siegen.
[1] R. Roskies and F. A. Schaposnik, Phys. Rev. D 23, 558 (1981); K. Furuya, R. E. Gamboa Saravi, and F. A. Schaposnik, Nucl. Phys. B208, 159 (1982).

[2] P. H. Damgaard, H. B. Nielsen, and R. Sollacher, Nucl. Phys. B385, 227 (1992); P. H. Damgaard and R. Sollacher, ibid. B433, 671 (1995).

[3] K. Fujikawa, Phys. Rev. Lett. 42, 1195 (1979); Phys. Rev. D 21, 2848 (1980); 23, 2262 (1981).

[4] E. Witten, Commun. Math. Phys. 92, 455 (1984).

[5] A. M. Polyakov and P. W. Wiegmann, Phys. Lett. 131B, 121 (1983); 141B, 223 (1984).

[6] E. Abdalla, M. C. B. Abdalla, and K. D. Rothe, Nonperturbative Methods in Two-Dimensional Quantum Field Theory (World Scientific, Singapore, 1991), p. 369.
[7] M. Wakimoto, Commun. Math. Phys. 104, 604 (1986).

[8] A. Gerasimov, A. Morozov, M. Olshanetsky, A. Marshakov, and S. Shastashvili, Int. J. Mod. Phys. A 5, 2495 (1990).

[9] K. D. Rothe, Nucl. Phys. B269, 269 (1986).

[10] A. N. Theron, F. A. Schaposnik, F. G. Scholtz, and H. B. Geyer, Nucl. Phys. B437, 187 (1995).

[11] F. G. Scholtz, A. N. Theron, and H. B. Geyer, Phys. Lett. B 345, 242 (1995).

[12] J. de Boer and L. Feher, Mod. Phys. Lett. A 11, 1999 (1996); "Wakimoto realization of current algebras: an explicit construction,"' hep-th/9611083.

[13] V. S. Dotsenko, Nucl. Phys. B338, 747 (1990).

[14] D. Bernard and G. Felder, Commun. Math. Phys. 127, 145 (1990). 